\title{
On the $k$-Mersenne-Lucas numbers
}

\author{
Mourad Chelgham $^{1}$ and Ali Boussayoud ${ }^{2}$ \\ ${ }^{1}$ LMAM Laboratory and Department of Mathematics \\ Mohamed Seddik Ben Yahia University, BP 98 Jijel, Algeria \\ e-mails: m.chelghameuniv-jijel.dz, chelghamm@yahoo.fr \\ ${ }^{2}$ LMAM Laboratory and Department of Mathematics \\ Mohamed Seddik Ben Yahia University, BP 98 Jijel, Algeria \\ e-mails: alibossayoudeuniv-jijel.dz, aboussayoudeyahoo.fr
}

Received: 9 April 2020

Revised: 1 December 2020

Accepted: 15 January 2021

Abstract: In this paper, we will introduce a new definition of $k$-Mersenne-Lucas numbers and investigate some properties. Then, we obtain some identities and established connection formulas between $k$-Mersenne-Lucas numbers and $k$-Mersenne numbers through the use of Binet's formula. Keywords: Mersenne numbers, Mersenne-Lucas numbers, $k$-Mersenne numbers, $k$-MersenneLucas numbers.

2010 Mathematics Subject Classification: 05E05, 11B39.

\section{Introduction}

For a long time, many researchers have studied well-known number sequences like Fibonacci, Lucas, Pell, Jacobasthal, and Mersenne in order to get intrinsic theory and applications of these numbers in many research areas as physics, engineering, architecture, nature, and art [5]. The generalizations of the sequences of numbers cited above have been defined in [1-4]. In this study, we are mainly interested by the Mersenne numbers.

Definition 1.1. For $n \in \mathbb{N}$, the Mersenne sequence, denoted by $\left\{M_{n}\right\}_{n \in \mathbb{N}}$, is defined recursively by

$$
\left\{\begin{array}{c}
M_{n}=3 M_{n-1}-2 M_{n-2}, \text { for all } n \geq 2 \\
M_{0}=0, M_{1}=1
\end{array} .\right.
$$


It is worth noting that Mersenne numbers belong to the same family as Fermat numbers, and thus, they share the same properties. Different studies have been carried out on these numbers and have been widely used in many recent real-world applications such as encryption, computer science and physics [6]. We defined $k$-Mersenne numbers as a generalization of the Mersenne numbers in [8].

Definition 1.2. For $n \in \mathbb{N}$, the $k$-Mersenne sequence, denoted by $\left\{M_{k, n}\right\}_{n \in \mathbb{N}}$, is defined recursively by

$$
\left\{\begin{array}{c}
M_{k, n}=3 k M_{k, n-1}-2 M_{k, n-2}, \text { for all } n \geq 2 \\
M_{k, 0}=0, M_{k, 1}=1
\end{array} .\right.
$$

The Binet's formula is given by

$$
M_{k, n}=\frac{r_{1}^{n}-r_{2}^{n}}{r_{1}-r_{2}}
$$

such that $r_{1}=\frac{3 k+\sqrt{9 k^{2}-8}}{2}$ and $r_{2}=\frac{3 k-\sqrt{9 k^{2}-8}}{2}$ are the roots of the characteristic equation of the sequence (1.1).

The terms of $k$-Mersenne sequence with a negative index are given by

$$
M_{k,-n}=\frac{-1}{2^{n}} M_{k, n}
$$

for all $n \geq 0$.

\section{The $k$-Mersenne-Lucas numbers and properties}

Depending on the definition of each of the $k$-Lucas, $k$-Pell-Lucas, $k$-Jacobsthal-Lucas and $k$-Mersenne numbers, we define new $k$-Mersenne-Lucas numbers, as follows.

Definition 2.1. For $n \in \mathbb{N}$, the $k$-Mersenne-Lucas numbers, denoted by $\left\{m_{k, n}\right\}_{n \in \mathbb{N}}$ are defined recursively by

$$
\left\{\begin{array}{c}
m_{k, n+1}=3 k m_{k, n}-2 m_{k, n-1}, \text { for all } n \geq 1 \\
m_{k, 0}=2, m_{k, 1}=3 k
\end{array} .\right.
$$

From relationship (2.1), we can write the first $k$-Mersenne-Lucas as follows:

$$
\left\{m_{k, n}\right\}_{n \in \mathbb{N}}=\left\{2,3 k, 9 k^{2}-4,27 k^{3}-18 k, 81 k^{4}-72 k^{2}+8, \ldots\right\} .
$$

The sequence $\left\{m_{k, n}\right\}_{n \in \mathbb{N}}$ has a recurrence relation of order 2 , its characteristic equation is given by

$$
r^{2}-3 k r+2=0
$$

whose roots are $r_{1}=\frac{3 k+\sqrt{9 k^{2}-8}}{2}$ and $r_{2}=\frac{3 k-\sqrt{9 k^{2}-8}}{2}$, such that

$$
r_{1}+r_{2}=3 k, \quad r_{1} r_{2}=2, \quad r_{1}-r_{2}=\sqrt{9 k^{2}-8} .
$$


Proposition 2.1 (Binet's formula). The $n$-th terms of k-Mersenne-Lucas numbers are given by

$$
m_{k, n}=r_{1}^{n}+r_{2}^{n}
$$

where $r_{1}, r_{2}$ are the roots of the characteristic equation (2.2) with $r_{1}>r_{2}$.

Remark 2.1. [7] Putting $k=1$ in Eq. (2.1), we obtain the definition of Mersenne-Lucas numbers $\left\{m_{n}\right\}_{n \in \mathbb{N}}$ as follows

$$
\left\{\begin{array}{c}
m_{n}=3 m_{n-1}-2 m_{n-2}, \text { for all } n \geq 2 \\
m_{0}=2, m_{1}=3
\end{array} .\right.
$$

The ratio of two successive numbers of $k$-Mersenne and $k$-Mersenne-Lucas numbers is

$$
\lim _{n \rightarrow \infty} \frac{m_{k, n+1}}{m_{k, n}}=\lim _{n \rightarrow \infty} \frac{M_{k, n+1}}{M_{k, n}}=r_{1}=\frac{3 k+\sqrt{9 k^{2}-8}}{2} .
$$

\section{Main results}

In this part, we give some results on $k$-Mersenne-Lucas numbers and established connection formulas between these numbers and $k$-Mersenne numbers.

Proposition 3.1. The $n$-th terms of k-Mersenne-Lucas numbers with a negative index are, as follows:

$$
m_{k,-n}=\frac{1}{2^{n}} m_{k, n}
$$

Proof. Replacing $n$ by $(-n)$ in Binet's formula we get the result. In particular, for $k=1$, we obtain the next result.

Corollary 3.0.1. [7] The Mersenne-Lucas numbers with negative index are given by $m_{-n}=\frac{1}{2^{n}} m_{n}$, for all $n \in \mathbb{N}$.

Theorem 3.1. For all $n, s \in \mathbb{N}$ and $k \geq 1$, the following identities are verified:

i) $m_{k, n} m_{k, s}+\left(9 k^{2}-8\right) M_{k, n} M_{k, s}=2 m_{k, n+s}$;

ii) $m_{k, n} m_{k, s}-\left(9 k^{2}-8\right) M_{k, n} M_{k, s}=2^{s+1} m_{k, n-s}$;

iii) $M_{k, s} m_{k, n}+M_{k, n} m_{k, s}=2 M_{k, n+s}$;

iv) $M_{k, n} m_{k, s}-M_{k, s} m_{k, n}=2^{s+1} M_{k, n-s}$.

Proof. By using Binet's formula of k-Mersenne and k-Mersenne-Lucas numbers and the fact that $r_{1} r_{2}=2$ and $r_{1}-r_{2}=\sqrt{9 k^{2}-8}$, we obtain all these identities.

Putting $n=s$ in $i$ ), ii) and iii) in the above theorem we obtain the next results. 
Corollary 3.1.1. For all $n \in \mathbb{N}$ and $k \geq 1$, the following identities are verified:

i) $m_{k, n}^{2}+\left(9 k^{2}-8\right) M_{k, n}^{2}=2 m_{k, 2 n}$;

ii) $m_{k, n}^{2}-\left(9 k^{2}-8\right) M_{k, n}^{2}=2^{n+2}$;

iii) $M_{k, n} m_{k, n}=M_{k, 2 n}$.

Theorem 3.2. For $n, s \in \mathbb{N}$ and $k \geq 1$, the following identities are verified:

i) $m_{k, n} m_{k, s}=m_{k, n+s}+2^{s} m_{k, n-s}$;

ii) $\left(9 k^{2}-8\right) M_{k, n} M_{k, s}=m_{k, n+s}-2^{s} m_{k, n-s}$;

iii) $m_{k, n} M_{k, s}=M_{k, n+s}-2^{s} M_{k, n-s}$;

iv) $m_{k, n+s} m_{k, n-s}=m_{k, n}^{2}+2^{n-s} m_{k, s}^{2}-2^{n+2} \quad$ (Catalan's identity);

v) $\left(9 k^{2}-8\right) M_{k, n+s} M_{k, n-s}=m_{k, 2 n}-2^{n-s} m_{k, 2 s}=m_{k, n}^{2}-2^{n-s} m_{k, s}^{2}$.

Proof. By the same method given in Theorem 3.1, the proof can be easily obtained.

Putting $n=s$ in $i$ ), ii) and $s=n+1$ et $s=1$ in iii) and $s=1$ in $i v$ ) and $v$ ) in the above theorem we obtain the next results.

Corollary 3.2.1. For all $n \geq 0$ and $k \geq 1$, the following identities hold:

i) $m_{k, n}^{2}=m_{k, 2 n}+2^{n+1}$;

ii) $\left(9 k^{2}-8\right) M_{k, n}^{2}=m_{k, 2 n}-2^{n+1}$;

iii) $M_{k, n+1} m_{k, n}=M_{k, 2 n+1}+2^{n}$ and $m_{k, n}=M_{k, n+1}-2 M_{k, n-1}$;

iv) $m_{k, n+1} m_{k, n-1}=m_{k, n}^{2}+\left(9 k^{2}-8\right) 2^{n-1} \quad$ (Cassini's identity);

v) $m_{k, n}^{2}=\left(9 k^{2}-8\right) M_{k, n+1} M_{k, n-1}+9 k^{2} 2^{n-1}$.

Theorem 3.3. (Sum of first terms of $\boldsymbol{k}$-Mersenne and $\boldsymbol{k}$-Mersenne-Lucas numbers) For all $n \in \mathbb{N}$ and $k>1$, the following identities are verified:
i) $\sum_{i=0}^{n} m_{k, i}=\frac{m_{k, n+1}-2 m_{k, n}+3 k-2}{3(k-1)}$ and $\sum_{i=0}^{n} M_{k, i}=\frac{M_{k, n+1}-2 M_{k, n}-1}{3(k-1)}$;
ii) $\sum_{i=0}^{n} m_{k, 2 i}=\frac{m_{k, 2(n+1)}-4 m_{k, 2 n}+9 k^{2}-6}{9\left(k^{2}-1\right)}$ and $\sum_{i=0}^{n} M_{k, 2 i}=\frac{M_{k, 2(n+1)}-4 M_{k, 2 n}-3 k}{9\left(k^{2}-1\right)}$;
iii) $\sum_{i=0}^{n} m_{k, 2 i+1}=\frac{m_{k, 2 n+3}-4 m_{k, 2 n+1}+3 k}{9\left(k^{2}-1\right)}$ and $\sum_{i=0}^{n} M_{k, 2 i+1}=\frac{M_{k, 2 n+3}-4 M_{k, 2 n+1}-3}{9\left(k^{2}-1\right)}$.

Proof. By Binet's formula of $k$-Mersenne and $k$-Mersenne-Lucas numbers and the fact that $r_{1} r_{2}=2, r_{1}+r_{2}=3 k$, one can easily prove that all these identities are verified. 
Corollary 3.3.1. For all $n \geq 0$, the following identities are verified:
i) $\sum_{i=0}^{n} m_{i}=2^{n+1}+n, \sum_{i=0}^{n} m_{2 i}=\frac{2^{2 n+2}+2}{3}+n$ and $\sum_{i=0}^{n} m_{2 i+1}=\frac{2^{2 n+3}+1}{3}+n$.
ii) $\sum_{i=0}^{n} M_{i}=2^{n+1}-n-2, \sum_{i=0}^{n} M_{2 i}=\frac{2^{2 n+2}-4}{3}-n$ and $\sum_{i=0}^{n} M_{2 i+1}=\frac{2^{2 n+3}-5}{3}-n$.

Proof. In this case $r_{1}=2$ and $r_{2}=1$. Using the fact that $\left\{2^{i}\right\}_{i \in \mathbb{N}}$ is a geometrical sequence such that $\sum_{i=0}^{n} 2^{i}=2^{n+1}-1$, these results can be easily verified.

Theorem 3.4. For all $n \in \mathbb{Z}$ and all $k \geq 1$, the following identity holds:

$$
A^{n}=M_{k, n} A-2 M_{k, n-1} I_{2},
$$

with $A=\left(\begin{array}{cc}3 k & -2 \\ 1 & 0\end{array}\right)$

Proof. By using the induction method on $n$ and the fact that $A^{n}=M_{k, n} A-2 M_{k, n-1} I_{2}$ and that $A^{-n}=M_{k,-n} A-2 M_{k,-n-1} I_{2}$ for all $n \in \mathbb{N}$, then we have

$$
\begin{aligned}
M_{k, n+1} A-2 M_{k, n} I_{2} & =\left(3 k M_{k, n}-2 M_{k, n-1}\right) A-2 M_{k, n} I_{2} \\
& =M_{k, n}\left(3 k A-2 I_{2}\right)-2 M_{k, n-1} A \\
& =\left(M_{k, n} A-2 M_{k, n-1} I_{2}\right) A=A^{n} . A=A^{n+1} .
\end{aligned}
$$

and

$$
\begin{aligned}
A^{-n-1} & =A^{-n} \cdot A^{-1}=\left(M_{k,-n} A-2 M_{k,-n-1} I_{2}\right) A^{-1} \\
& =\left(\frac{-1}{2^{n}} M_{k, n} A-2 \frac{-1}{2^{n+1}} M_{k, n+1} I_{2}\right) A^{-1}=\left(\frac{-1}{2^{n}} M_{k, n} I_{2}+\frac{1}{2^{n}} M_{k, n+1} A^{-1}\right) .
\end{aligned}
$$

Since $A^{-1}=\frac{-1}{2}\left(A-3 k I_{2}\right)$, then,

$$
\begin{aligned}
A^{-n-1} & =-\frac{1}{2^{n+1}} M_{k, n+1} A+\frac{1}{2^{n+1}}\left(3 k M_{k, n+1}-2 M_{k, n}\right) I_{2} \\
& =-\frac{1}{2^{n+1}} M_{k, n+1} A-2 \frac{-1}{2^{n+2}} M_{k, n+2} I_{2}=M_{k,-n-1} A-2 M_{k,-n-2} I_{2} .
\end{aligned}
$$

This completes the proof.

Theorem 3.5. For all $n \in \mathbb{Z}$ and all $k \geq 1$, the following identity holds:

$$
A^{n}=\left(\begin{array}{cc}
M_{k, n+1} & -2 M_{k, n} \\
M_{k, n} & -2 M_{k, n-1}
\end{array}\right)
$$

with $A=\left(\begin{array}{cc}3 k & -2 \\ 1 & 0\end{array}\right)$.

Proof. It has been shown in [8] that for all integer $n \geq 0$ this identity holds. We need to show that the above equality holds for $n \in \mathbb{Z}_{-}$, that is $A^{-n}=\left(\begin{array}{cc}M_{k,-n+1} & -2 M_{k,-n} \\ M_{k,-n} & -2 M_{k,-n-1}\end{array}\right)$ for all $n \geq 0$. 
From the above theorem we have $A^{-n}=M_{k,-n} A-2 M_{k,-n-1} I_{2}$. By replacing $A$ and $I_{2}$ we have

$$
A^{-n}=\left(\begin{array}{cc}
3 k M_{k,-n}-2 M_{k,-n-1} & -2 M_{k,-n-1} \\
M_{k,-n} & -2 M_{k,-n-1}
\end{array}\right)=\left(\begin{array}{cc}
M_{k,-n+1} & -2 M_{k,-n} \\
M_{k,-n} & -2 M_{k,-n-1}
\end{array}\right) .
$$

Hence for all $n \in \mathbb{Z}$ the equality holds. This completes the proof.

Theorem 3.6. For all $n \in \mathbb{N}$ and $k \geq 1$, the following identity is verified:

$$
B^{n}=\left(\begin{array}{cc}
\frac{m_{k, n}}{2} & \frac{\left(9 k^{2}-8\right) M_{k, n}}{2} \\
\frac{M_{k, n}}{2} & \frac{m_{k, n}}{2}
\end{array}\right) \text {, with } B=\left(\begin{array}{cc}
\frac{3 k}{2} & \frac{9 k^{2}-8}{2} \\
\frac{1}{2} & \frac{3 k}{2}
\end{array}\right) \text {. }
$$

Proof. Suppose that the above identity is verified for $n \geq 2$. We have

$$
\begin{aligned}
B^{n+1} & =\left(\begin{array}{cc}
\frac{m_{k, n}}{2} & \frac{\left(9 k^{2}-8\right) M_{k, n}}{2} \\
\frac{M_{k, n}}{2} & \frac{m_{k, n}}{2}
\end{array}\right)\left(\begin{array}{cc}
\frac{3 k}{2} & \frac{9 k^{2}-8}{2} \\
\frac{1}{2} & \frac{3 k}{2}
\end{array}\right) \\
& =\frac{1}{4}\left(\begin{array}{cc}
3 k m_{k, n}+\left(9 k^{2}-8\right) M_{k, n} & \left(9 k^{2}-8\right)\left(3 k M_{k, n}+m_{k, n}\right) \\
3 k M_{k, n}+m_{k, n} & 3 k m_{k, n}+\left(9 k^{2}-8\right) M_{k, n}
\end{array}\right) .
\end{aligned}
$$

It easy to show that $3 k M_{k, n}+m_{k, n}=2 M_{k, n+1}$ and $3 k m_{k, n}+\left(9 k^{2}-8\right) M_{k, n}=2 m_{k, n+1}$. Therefore,

$$
B^{n+1}=\frac{1}{4}\left(\begin{array}{cc}
2 m_{k, n+1} & 2\left(9 k^{2}-8\right) M_{k, n+1} \\
2 M_{k, n+1} & 2 m_{k, n+1}
\end{array}\right)=\left(\begin{array}{cc}
\frac{m_{k, n+1}}{2} & \frac{\left(9 k^{2}-8\right) M_{k, n+1}}{2} \\
\frac{M_{k, n+1}}{2} & \frac{m_{k, n+1}}{2}
\end{array}\right) .
$$

This completes the proof.

Remark 3.7. All the obtained results in the above theorems and corollaries are verified for $k=1$, except those in Theorem 3.3.

Theorem 3.8. For $n \in \mathbb{N}$, the new generating function of the $k$-Mersenne-Lucas sequence is given by

$$
\sum_{n=0}^{\infty} m_{k, n} t^{n}=\frac{2-3 k t}{1-3 k t+2 t^{2}} .
$$

Proof. The ordinary generating function associated is defined by

$$
\begin{aligned}
\sum_{n=0}^{\infty} m_{k, n} t^{n} & =m_{k, 0}+m_{k, 1} t+\sum_{n=2}^{\infty} m_{k, n} t^{n} \\
& =m_{k, 0}+m_{k, 1} t+\sum_{n=2}^{\infty}\left(3 k m_{k, n-1}-2 m_{k, n-2}\right) t^{n} \\
& =m_{k, 0}+m_{k, 1} t+3 k \sum_{n=2}^{\infty} m_{k, n-1} t^{n}-2 \sum_{n=2}^{\infty} m_{k, n-2} t^{n} \\
& =m_{k, 0}+\left(m_{k, 1}-3 k m_{k, 0}\right) t+3 k t \sum_{n=0}^{\infty} m_{k, n} t^{n}-2 t^{2} \sum_{n=0}^{\infty} m_{k, n} t^{n} .
\end{aligned}
$$

And we can easily deduce the new generating function of the $k$-Mersenne-Lucas sequence by reordering the obtained result. This completes the proof. 
Remark 3.9. [7] Setting $k=1$ in the Eq. (3.1) yields the generating function of the MersenneLucas sequence.

\section{Acknowledgements}

The authors would like to thank the anonymous referees for their valuable comments and suggestions. This work was supported by Directorate General for Scientific Research and Technological Development (DGRSDT), Algeria.

\section{References}

[1] Catarino, P. (2013). On some identities and generating functions for $k$-Pell numbers. International Journal of Mathematics and Analysis, 7(38), 1877-1884.

[2] Catarino, P. (2014). On some identities and generating function for $k$-Fibonacci sequence. International Journal of Contemporary Mathematical Sciences, 9(1), 37-42.

[3] El-Mikkawy, M., \& Sogabe, T. (2010). A new family of $k$-Fibonacci numbers, Applied Mathematics and Computation, 215(12), 4456-4461.

[4] Falcon, S. (2011). On the $k$-Lucas numbers. International Journal of Contemporary Mathematical Sciences, 6(21), 1039-1050.

[5] Koshy, T. (2001). Fibonacci and Lucas numbers with applications, John Wiley \& Sons.

[6] Koshy, T., \& Gao, Z. (2013). Catalan numbers with Mersenne subscripts. Mathematical Scientist, 38, 86-91.

[7] Saba, N., Boussayoud, A. \& Kanuri, K.V.V. (2021). Mersenne Lucas numbers and complete homogeneous symmetric functions. Journal of Mathematics and Computer Science, 24(2), $127-139$.

[8] Uslu, K., \& Deniz, V. (2017). Some identities of $k$-Mersenne numbers. Advances and Applications in Discrete Mathematics, 18(4), 413-423. 